\title{
Impact of surface electric properties of carbon-based thin films on platelets activation for nano-medical and nano-sensing applications
}

\author{
Varvara Karagkiozaki ${ }^{1,2}$ \\ Stergios Logothetidis' \\ Sylvie Lousinian' \\ George Giannoglou ${ }^{2}$ \\ 'Physics Department, Laboratory \\ for Thin Films - Nanosystems \\ and Nanometrology (LTFN); ${ }^{2}$ Medical \\ School, AHEPA University General \\ Hospital, I st Cardiology Department, \\ Aristotle University of Thessaloniki, \\ Greece
}

\begin{abstract}
Electric surface properties of biomaterials, playing key role to various biointerfacial interactions, were related to hemocompatibility and biosensing phenomena. In this study, the examination of surface electric properties of amorphous hydrogenated carbon thin films (a-C:H) was carried out by means of electrostatic force microscope (EFM) and observation of differences in spatial charge distribution on the surface of the examined films during platelets adhesion was made. The thrombogenic potential of a-C:H thin films developed by magnetron sputtering with $\sim 42 \% \mathrm{sp}^{3}$ content and hydrogen partial pressure during deposition was evaluated, by in situ observation with atomic force microscope (AFM) of platelets' activation and their subsequent adhesion. Platelet-rich plasma drawn from healthy donors was used and semi-contact mode of AFM was applied. Platelets behavior and their correlation with the electric surface properties of the examined a-C:H films by EFM was made for hemocompatibility enhancement and sensing platelets that are less electrical negatively charged and with higher tendency to aggregate and form thrombus. The results are discussed in view of the effect of different deposition conditions of hydrogenated carbon films on their structural and morphological characteristics, surface roughness and electrical properties attributing to different hemocompatibility and sensing aspects.
\end{abstract}

Keywords: amorphous hydrogenated carbon, platelets, thrombogenicity, atomic force microscopy, electric force microscopy, medical applications

\section{Introduction}

The hemocompatibility of an artificial material in the body is an extremely complicated and multiparametric process that determines the efficacy and longevity of biomedical implants. During the past decades, research effort has focused on the parameters that affect the host response to biomaterials in order to improve their behavior for medical applications such as biosensors, vascular prostheses, etc.

The surface properties of the implant strongly influence the properties of the layer and this influence needs to be understood and controlled in order to optimize biocompatibility. Although various studies have focused on the effect of chemical, physical and topographical properties of biomaterials on hemocompatibility, the role of electrical field distribution on their surface and its relation with other surface and morphological characteristics has not yet been investigated extensively.

Coronary heart disease (CHD) leading to myocardial infarction and other undesirable complications has a basic pathogenic mechanism, which is thrombus formation within the lumen of coronary arteries, whose endothelial surfaces facilitate platelet aggregation. Recent studies have shown that patients with CHD have a significantly higher proportion of platelets with low densities and less electrical negative charges onto their surfaces representing platelets that have a high tendency 
to aggregate (Mandic et al 2002). The potential application of surface-electrical charged nanomaterials made by ionbombardment techniques (Weng et al 2002; Laskarakis et al 2004) as nanosensors for the detection of platelets which are prone to activation and aggregation leading to the obstruction of arteries has not been examined yet.

When a biomaterial is implanted in the human body, water and protein adsorption to surfaces typically occurs. Then, cells such as leucocytes and platelets contact biomaterial surfaces and are activated due to stimulation by cytokines and growth factors (Hussain et al 2004; Massa et al 2005). A common failure of a biomaterial arises from thrombogenesis, which is initiated by platelets' adhesion onto artificial surface and activation. Thus, a significant factor that determines hemocompatibility, is the platelets' response to the surface of a biomaterial, as indicated by their morphological changes (Yang 2003; Plant et al 2007).

Several studies reported about the biocompatibility of diamond-like carbon and of amorphous hydrogenated carbon (a-C:H) coatings (Cui et al 2000; Hauert et al 2003; Vinnichenko et al 2004; Maguire et al 2005). Carbon-based thin films with an increased fraction of $\mathrm{sp}^{3}$ bonds are known to possess high-mechanical hardness, low surface roughness, chemical inertness, and exhibit good blood compatibility (Logothetidis et al 1999; Charitidis et al 2000; Robertson 2002). In addition, carbon layers developed onto polytetrafluoroethylene were observed to drastically increase adhesion and proliferation of human endothelial cells, which is beneficial for stent applications (Kubová et al 2007).

This aspect is interesting for the improvement of biomedical devices and biosensors. In a previous work of our research team, it was shown that a-C:H thin films, developed with $\mathrm{rf}$ reactive magnetron sputtering, with $\mathrm{sp}^{3}$ content of $\sim 40 \%$ and small amount of $\mathrm{H}_{2}$ in plasma during deposition, exhibited good hemocompatibility in terms of protein adsorption as shown in Table 1. More 1, the ratio of the human serum albumin to fibrinogen surface concentration, which is used as an indication of the hemocompatibility (reduced thrombogenicity potential), was evaluated to be higher for the aforementioned a-C:H thin films (Logothetidis et al 2005; Lousinian et al 2007). This conclusion has led to the need of investigating further the a-C:H thin films in relation with platelets adhesion and activation and of correlating it with their electrical and surface properties not only for the design of more biocompatible implants but also for the development of biosensors for detection of platelets culprits for thrombosis.

Atomic force microscopy (AFM) has been implemented as a surface characterization technique for the investigation of nanotopography of a-C:H thin films (Liu et al 2006; Zhu et al 2006). This nanotool has the ability to resolve features at atomic dimensions providing the advantage to observe and analyze the kinetics of biointerface interactions at nanoscale, such as the phenomena of human platelets adhesion onto a-C:H thin films. Furthermore, the electrostatic force microscope (EFM) based on AFM principles was used to detect the electric fields' distribution on the surface of the sample with submicron resolution. EFM images contain qualitative information about the electric properties of the samples (Hai-Tong et al 2007). In this work, a methodology was developed for evaluating the thrombogenicity of biomaterials by AFM and for assessing the electrical behavior of materials by EFM.

\section{Experimental methods}

Amorphous hydrogenated carbon thin films were deposited on c-Si (100) wafer by RF reactive magnetron sputtering in a high vacuum chamber with $20 \% \mathrm{H}_{2}$ as the reactive gas; one series of a-C:H films were deposited with bias voltage $-40 \mathrm{~V}$ applied onto the substrate (Biased) and the second with no

Table I The sample codes deposition parameters, refractive index, $\mathrm{sp}^{3}$ content for the a-C:H thin films and albumin/fibrinogen ratio as measured by spectroscopic ellipsometry in the energy region 1.5-6.5 eV, in previous work of our lab (Logothetidis et al 2005)

\begin{tabular}{llllll}
\hline Sample code & $\mathbf{V}_{\mathbf{b}}($ Volt $)$ & $\mathbf{H}_{\mathbf{2}}$ at \% in plasma & Refractive index, $\boldsymbol{n}$ & sp $^{3}$ fraction (\%) & HSA/Fib ratio \\
\hline \#BI & -40 & 5 & 1.683 & $40-44$ & 0.276 \\
\#B2 & -40 & 10 & 1.679 & $40-44$ & 0.274 \\
\#B3 & -40 & 20 & 1.634 & $40-44$ & 0.216 \\
\#FI & +10 & 10 & 1.488 & $40-44$ & 0.93 \\
\#F2 & +10 & 20 & 1.501 & $40-44$ & 1.02 \\
\#F3 & +10 & 30 & 1.498 & $40-44$ & 0.396 \\
\#F4 & +10 & & 1.529 & $40-44$ & 0.567
\end{tabular}

Abbreviations: \#BI, 2, biased samples; \#FI, 2, floating samples; $\mathrm{V}_{\mathrm{b}}$, bias voltage; HSA, human serum antigen; Fib, fibrinogen. 
substrate bias (Floating). The Si-100 substrates which were n-type (phosphorous doped) with 1-5 Ohm-cm specific resistance, were ultrasonically cleaned in tetrachloroethylene, acetone, and methanol at $60{ }^{\circ} \mathrm{C}$ (Logothetidis 2002). For the conduction of the experiment, human platelet-rich plasma (PRP) was prepared after the centrifugation of whole blood at $800 \mathrm{rpm}$, at room temperature (RT). The collecting time was 7-10 minutes. The blood was drawn by venopuncture from healthy donors, who did not take medication such as aspirin or clopidogrel, which affect platelets function, and then kept in tubes with $3.8 \%$ citrate acid. After centrifugation, the PRP was diluted with homologous plasma in a proportion of $1: 1000$, in order to achieve a blood platelet density of $330 / \mathrm{mm}^{3}$. Afterwards, the a-C:H thin films were cleaned by $\mathrm{N}_{2}$ air flow for the removal of any contaminants and then they were incubated in PRP at RT and examined by AFM and EFM. Measurements were performed with SOLVER P47H Scanning Probe Microscope (NT-MDT; NTI Instruments, Moscow, Russia; NT-MDT 2008) in ambient environment. Standard "Golden" silicon cantilevers with nominal spring constant $11.5 \mathrm{~N} / \mathrm{m}$ and resonance frequency $255 \mathrm{kHz}$ and high aspect ratio conical tips (cone angle less than $22^{\circ}$ ) with curvature radius of $10 \mathrm{~nm}$ and tip height of 10-20 $\mu \mathrm{m}$, were used. Semi-noncontact operation mode of AFM was employed to avoid the damage of the soft platelets by the sharp conical tip (Uchihashi et al 2000). The platelets morphology was not changed during AFM scanning as they were fixed with $5 \mu$ l glutaraldehyde $1 \%$ for one and two hours of observations and were not fully dehydrated.

The morphology of the adherent platelets was investigated at one and two hours intervals and ten fields were chosen at random to obtain statistical averages (by x-square statistical method) of the adherent platelets The evaluation of surface roughness of the plain films and during platelets adhesion was made by measurements of peak to valley distance (peak-to-peak), and root-mean-square roughness $\left(\mathrm{R}_{\mathrm{rms}}\right)$ parameters.

The EFM mode, based on the same principles as AFM, maps electric properties on a sample surface by measuring the electrostatic force between the surface and a biased AFM cantilever that applies a voltage between the tip and the sample. The cantilever deflects when it scans over static charges, and the interaction of the probe-sample system, such as strength of interaction, amplitude and phase of oscillations of the probe, gives information about electric properties of the sample. EFM measurements are based on a double-pass technique: i) during the first pass, the surface relief is traced in the region where the short-range van der
Waals forces are dominant, whereas ii) during the second pass, the biased cantilever is raised to a given height, in the region where the strong long-range electrostatic forces prevail. The only source of the signal change will be the change of the electrostatic forces and one of the following signals is acquired:

a) amplitude of oscillations of a cantilever (MAG),

b) phase shift between a piezoelectric oscillator and a cantilever (PHASE),

c) product of amplitude of oscillations of a cantilever and sin of phase shift (MAG*SIN),

d) product of amplitude of oscillations of a cantilever and cosine of phase shift (MAG*COS).

These parameters can provide qualitative information of electrical field distribution on a surface and not a quantitative one and are useful for comparing samples with different electrical charged sufaces.

In this work, the applied voltage (BV) between the probe and the sample was (+) 7.5 voltages whereas the distance $(\Delta \mathrm{Z})$ between vertical positions of the scanner during the first and the second pass was $10 \mathrm{~nm}$. The phase and MAG*COS signals were used to interpret the information on the electrical distribution on the surfaces of a-C:H thin films.

\section{Results and discussion}

The hemocompatibility study of the a-C:H thin films used in this work has shown that the films deposited under floating conditions present much better hemocompatible behavior than the ones deposited under application of negative bias voltage (Mitsakakis et al 2007).

Table 2 presents details about mean values of peak to peak distance and root mean square roughness parameters with their standard deviations for ten randomly scanned AFM areas of platelets incubation times: 0 min (bare substrate), 1 hour, and 2 hours. These results derived from AFM analysis of the whole area of the ten fields with length scale of $10 \times 10 \mu \mathrm{m}$. By comparing these values for 'bare' a-C:H substrates through AFM images (Figures 1a, 1b) it is clearly deduced that the floating ones exhibit higher surface roughness $(2.45 \mathrm{~nm})$ than the biased ones $(0.8 \mathrm{~nm})$ and this fact can be attributed to the different conditions of deposition. As the sputtered carbon atoms under the influence of bias voltage have higher kinetic energy, a layer-by-layer growth occurs comparing with the island one characterized the deposition without bias substrate, leading to the lower roughness of the biased thin films.

Platelets, in their resting stage, are disc shaped in size of $0.5-3 \mu \mathrm{m}$ in order to resist blood pressure and avoid 
Table 2 The film types, incubation times of platelets (PLTs) and morphology parameters of surfaces, including the mean values of peak-to-peak distance, RMS roughness and their standard deviation values, as measured for ten randomly scanned AFM areas

\begin{tabular}{llll}
\hline Film type & Incubation time (hours) & Mean peak-to-peak $(\mathbf{n m})$ & Mean RMS roughness, $\mathbf{R}_{\text {rms }}$ (nm) \\
\hline a-C:H (biased) & 0 & $14.8 \pm 1.2$ & $0.8 \pm 0.1$ \\
PLTs/a-C:H (biased) & 1 & $658.9 \pm 71$ & $93 \pm 6.3$ \\
PLTs/a-C:H (biased) & 2 & $813 \pm 83$ & $138.9 \pm 5.8$ \\
a-C:H (floating) & 0 & $39 \pm 5.7$ & $2.45 \pm 0.7$ \\
PLTs/a-C:H (floating) & 1 & $591.4 \pm 79.9$ & $89.9 \pm 20.42$ \\
PLTs/a-C:H (floating) & 2 & $626.3 \pm 74$ & $95.3 \pm 19.4$ \\
\hline
\end{tabular}

Abbreviations: a-C:H, amorphous hydrogenated carbon; PLTs, platelets.

sticking together. When they come in contact with an artificial material and activate, their shape become flatter, their granules are gathered into the center of the cell contributing to the composition of pseudonucleus (egg-like type) and broad lamellae are extended from the cells' periphery. The elaboration of filopods is essential for their spread onto the surfaces and their aggregation. Besides morphological changes, platelets release agonist compounds of dense and a-granules to attract and facilitate the recruitment of additional platelets and leucocytes promoting the growth of platelet thrombus onto the artificial material. (Hartwig 2007; Oberprieler 2007).

(a)
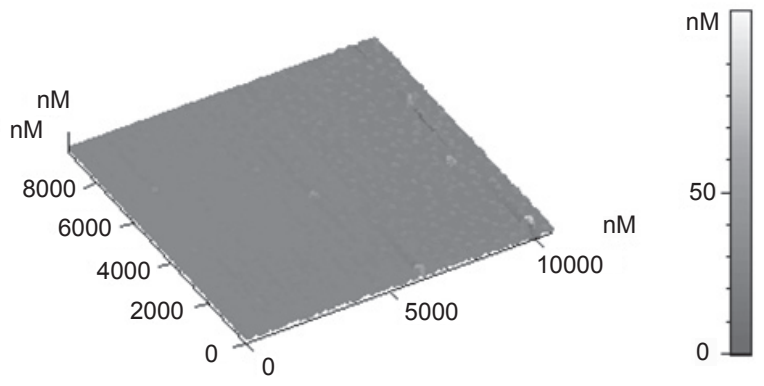

(b)

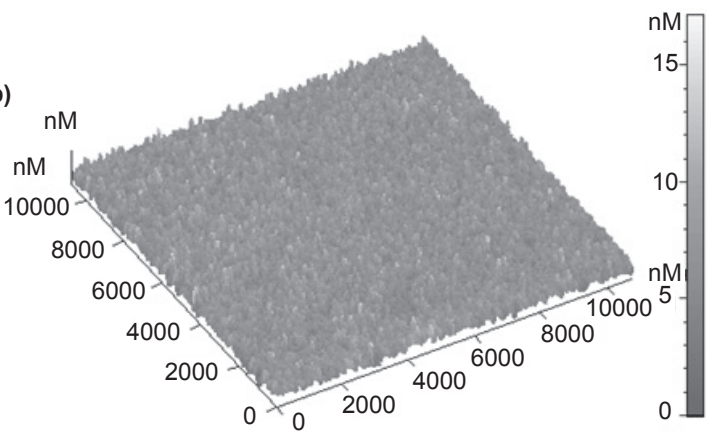

Figure I Atomic force microscope topography image $(10 \mu \mathrm{m} \times 10 \mu \mathrm{m})$ of (a) a-C: $\mathrm{H}$ (Biased) as deposited, (b) a-C:H (Floating) as deposited.
All these morphological characteristics of platelets during their activation were taken into account for the analysis of AFM images. By observing the AFM images of platelets adhering onto a-C:H thin films, it can be deduced that on the Floating carbon thin films after 1 and $2 \mathrm{~h}$ of incubation, the platelets undergo alterations in morphology during activation and they form clusters. It can be noticed in Figure 2a., that after 1hour incubation-time of platelets on Floating carbon films with size of $10 \times 10 \mu \mathrm{m}$, platelets appear to aggregate in height and then after two hours this core of platelets' cluster increase in height as well as in length, developing a structure like an 'island' (shown in Figure 2b, with image size of: $21 \times 21 \mu \mathrm{m}$ ).

(a)

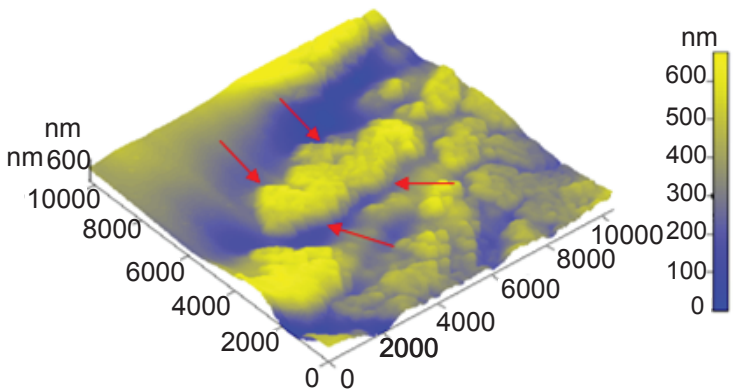

(b)
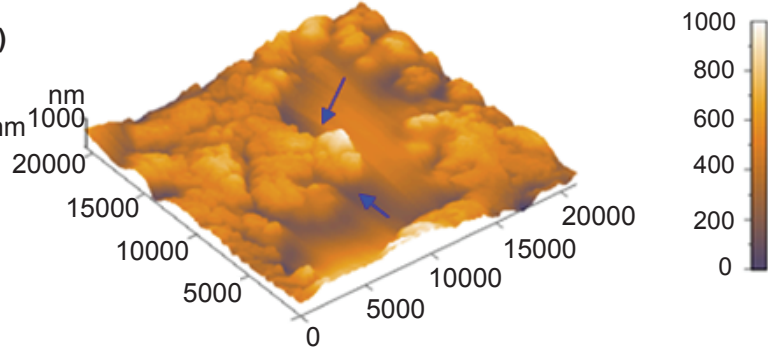

Figure 2 Atomic force microscope topography image of (a) platelets on a-C:H (Floating) after I hour incubation time (scan size $10 \mu \mathrm{m} \times 10 \mu \mathrm{m}$ ). Arrows indicate the platelets' clusters. (b) Platelets on a-C:H (Floating) after 2 hours incubation time, with a size of $20 \mu \mathrm{m} \times 20 \mu \mathrm{m}$. Arrows indicate the formation of platelets' cluster like an island. 
Estimation of surface roughness of platelets via the measurements of mean peak-to peak and $\mathrm{R}_{\mathrm{rms}}$ parameters, shown in Figures 3 and 4, indicates that there is a statistically significant time-dependant increase of its values $(\mathrm{p}<0.001)$. This can be attributed to platelets' gradual aggregation and the increase of the height of their clusters during time.

In contrast, on the biased carbon films, platelets have a higher tendency to aggregate and this can be easily noticed by comparing the AFM images after 1 and 2 hours incubation time with the images of floating platelets. This fact can be verified by the comparative diagram of $\mathrm{R}_{\mathrm{rms}}$ and peak-to-peak parameters versus platelets incubation time (as shown in Figures 3 and 4). It is also observed in AFM topography images (Figure 5), that biased a-C:H thin films like floating platelets, after one hour of platelets incubation, form clusters increasing in height and after two hours of study, they present the 'island' type formation. Thus, this model of platelets aggregation can be suggested for the evaluation of thrombogenicity of biomaterials.

The aggregation of platelets is an important step in the process of formation of a clot, which occurs when inactivated clotting factors are enzymically activated by procoagulant complexes that are bound onto platelet membranes. As a result, the insoluble fibrin polymer is formed, which encapsulates the platelets and produces a thrombus. Thus, the thrombogenicity of the material is increased and becomes less biocompatible. All the aforementioned quantitative data show that the floating a-C:H thin films exhibit less thrombogenicity than the ones with the appliance of ion bombardment during deposition, attributing partially to the higher surface roughness of the former ones. Recent studies show that the smooth surface may promote thrombosis and that real-time adsorption of albumin (HSA) and fibrinogen

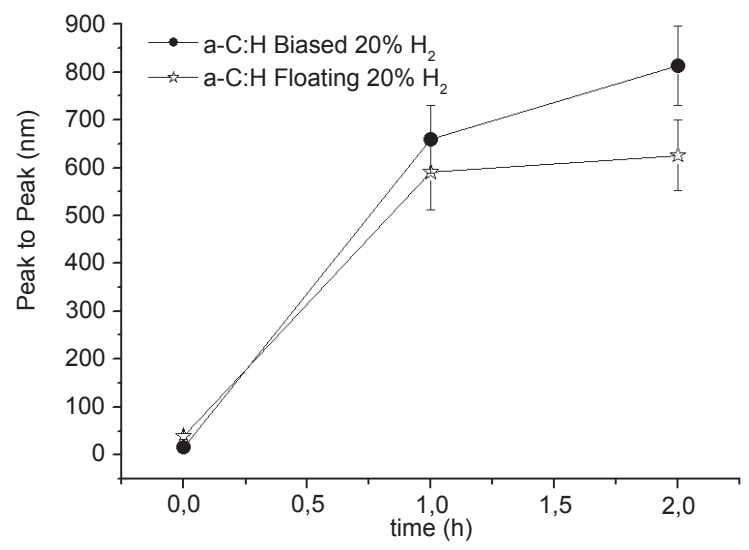

Figure 3 Comparative diagram of mean Peak-to Peak parameter of examined platelets versus incubation time for the two different types of a-C:H thin films.
(Fib) study by spectroscopic ellipsometry and AFM onto floating and biased carbon thin films indicate that the highest HSA/Fib ratio, as an index of better hemocompatibility, was observed at floating a-C:H thin films (Logothetidis 2007).

The knowledge of the electrical properties of biomaterials at the nanometer scale is essential as the involvement of electron exchange can occur in proteins adsorption and platelets adhesion process from the physiological media. This electron transfer may induce conformation changes of adsorbed proteins that act as chemoattractants for platelets and cause their degeneration whereas the electrical charge of the artificial materials is considered to contribute to the activation of coagulant system and of the cellular response (Shih et al 2003). The electric charge property of blood was reported by Abramson (1928) when he studied the properties of human lymphocytes and proposed that the components of the blood are negatively charged. Platelets are also regarded to have negative charges onto their surfaces due to the presence of sialic acid (Nacetyl-neuraminic acid), a negatively charged sugar and to a much lesser extent negatively charged amino acids like glutamate and aspartate into their membranes. This fact helps cells to keep a distance from each other due to repulsion of the same negative charges inhibiting their aggregation (Bosmann 1972).

Recent studies have shown that patients with CHD compared to healthy controls have surprisingly more small platelets having the lowest amount of sialic acid into their membranes. The lower sialic acid content in platelets from CHD patients could represent a contributing factor for the higher aggregability of these platelets, since a lower negative surface charge could result in less repulsion and higher likelihood of aggregation (Lupu et al 1988; Trip et al 1990; Kotze et al 1993; Opper 1995). Therefore, platelet sialic

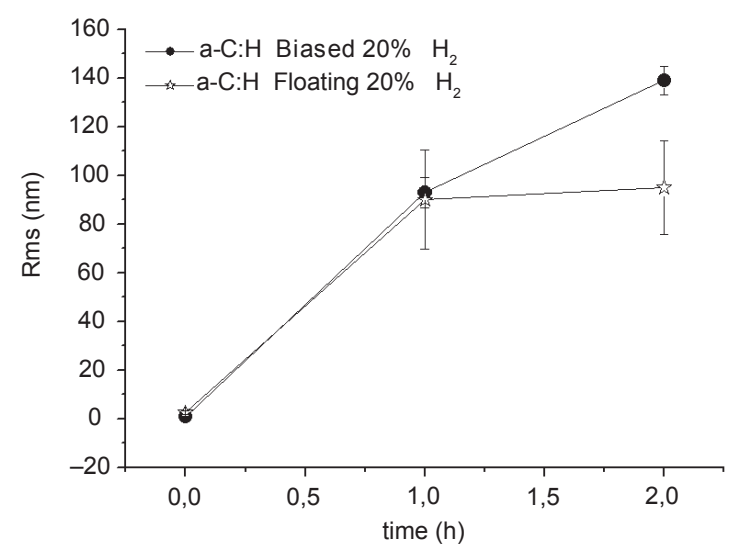

Figure 4 Comparative diagram of mean RMS roughness of examined platelets versus incubation time for the two different types of a-C:H thin films. 
acid potentially represents a pathogenic factor for thrombus formation and subsequent acute coronary syndromes such as myocardial infarction. At this point, the potential application of the examined nanofilms as sensors for the detection of platelets that are prone to aggregate and cause thrombosis, is essential either as screening test for CHD, for differential diagnosis of acute chest pain, or as stent coatings with sensing capability. Further steps on this work include verification of the above results and the development of biosensors based on these nanomaterials for sensing platelets that are prone to aggregate.

As blood-clotting is a charge-transferring process, the thrombogenicity of the examined a-C: $\mathrm{H}$ thin films was estimated in regards of electrical surface distribution. Figure 6a illustrates the EFM image and especially the $\mathrm{MAG}^{*} \mathrm{COS}$ signal of platelets adhering onto floating a-C:H thin films. As the applied voltage (BV) between the probe and the sample was $(+) 7.5 \mathrm{~V}$, when the positively charged tip meets the surface of the sample negative charges, attractive electrostatic forces between the tip and the sample were applied whereas when it meets positive charges,

(a)

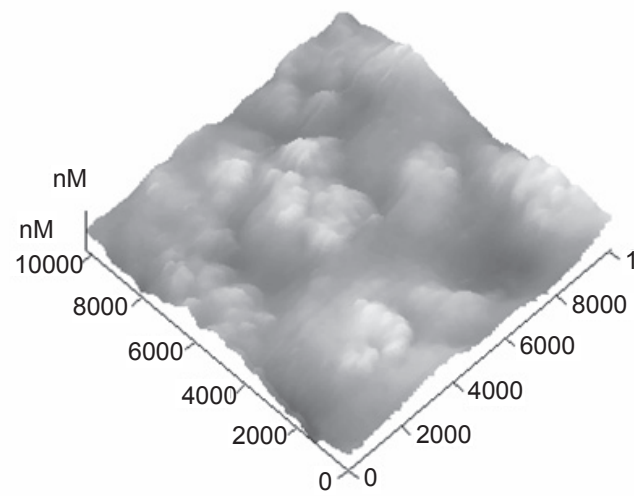

(b)

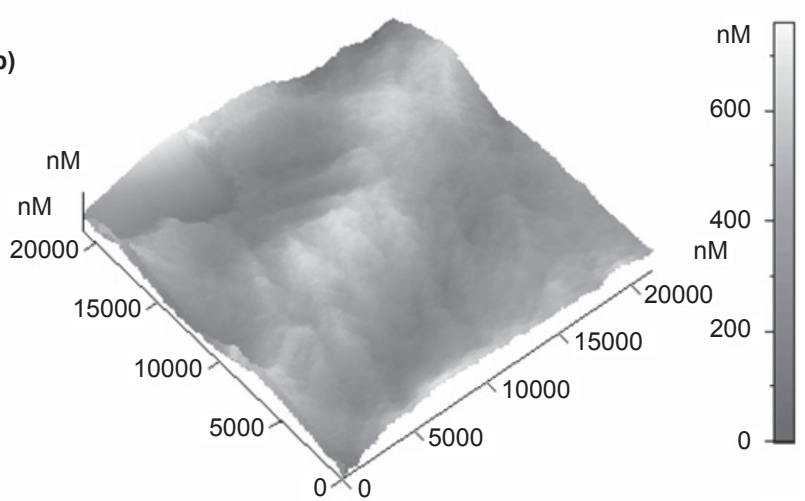

Figure 5 Atomic force microscope topography image of (a) platelets on a-C:H (Biased) after I hour incubation time (scan size $10 \mu \mathrm{m} \times 10 \mu \mathrm{m})(\mathbf{b})$ Platelets on a-C:H (Biased) after 2 hours incubation time, with a size of $20 \mu \mathrm{m} \times 20 \mu \mathrm{m}$. repulsive forces were assessed. By observing the EFM image in Figures $6 \mathrm{a}$ and $6 \mathrm{~b}$, it can be realized that $\mathrm{MAG}^{*} \mathrm{COS}$ and phase have negative values, implying that there are attractive forces between platelets and the positively charged tip leading to the conclusion that platelets are electrically negative. More precisely, by performing X-cross section at $4500 \mathrm{~nm}$ of the relevant AFM topography image (Figures 7a, 7b) at the lower boundaries of the central platelets' cluster (black circle) it is obvious (as shown with the red arrow) that the red circle in Figure 7a is indicative of a platelet area with $250 \mathrm{~nm}$ height whereas the $\mathrm{x}$-section of the same image (Figure 7c) at $7200 \mathrm{~nm}$ which indicates the upper boundaries of the same platelets' cluster the red circle in Figure 7a shows an area with height of $370 \mathrm{~nm}$. By comparing these two X-sections of the lower and upper boundaries of the central platelets' cluster respectively, there is a difference in height of approximately $120 \mathrm{~nm}$. By observing the EFM image in Figure 6a it can be seen that the lower boundaries of the examined platelets aggregation are blue implying that they are more electrically negative compared with the areas without platelets (as shown by green square). In contrast the upper boundaries

(a)

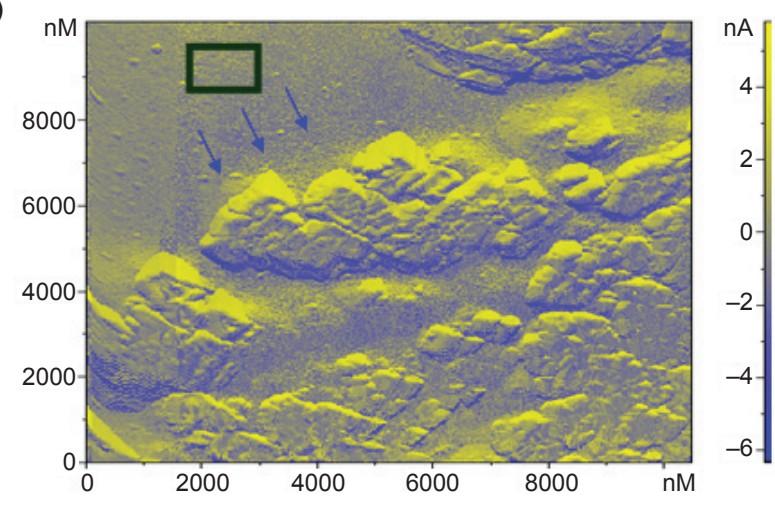

(b)

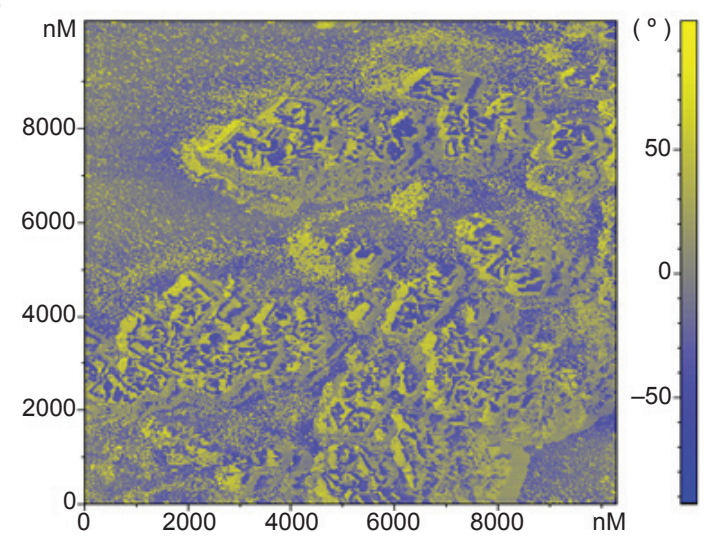

Figure 6 Electric force microscope image: platelets on a-C:H (Floating) after I hour incubation time (scan size $10 \mu \mathrm{m} \times 10 \mu \mathrm{m}$ ) (a) MAG*COS. The blue arrows indicate the platelets' clusters whereas the green square denotes an area free of platelets (b) Phase. 
(a)

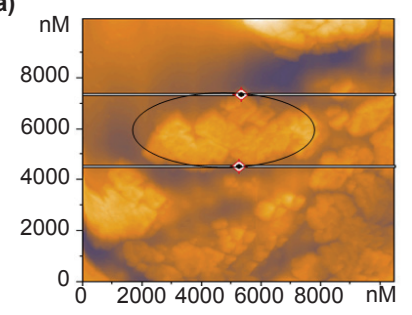

(b)

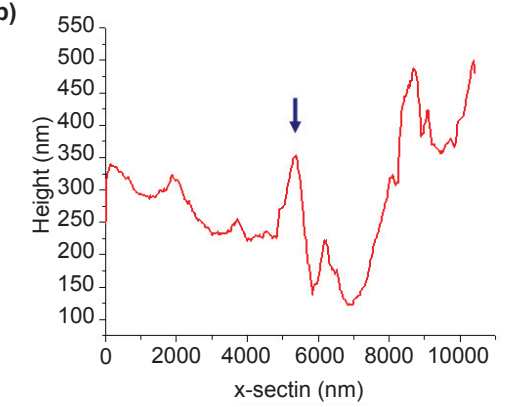

(c)
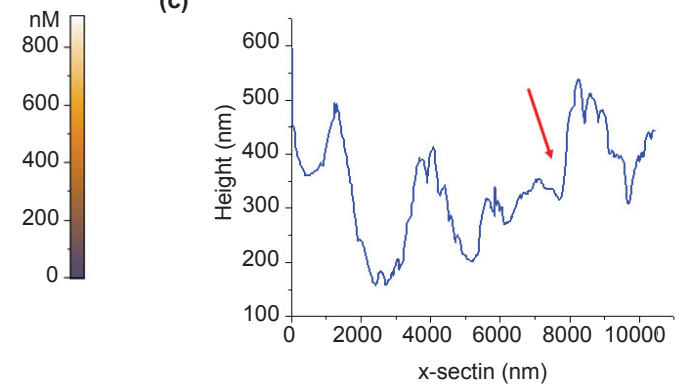

Figure 7 (a) Atomic force microscope topography image of platelets on a-C:H (Floating) after I hour incubation time (scan size $10 \mu \mathrm{m} \times 10 \mu \mathrm{m})$. Black circle indicates the platelets' cluster.The two lines indicate the upper and lower boundaries of this platelets cluster where the $\mathrm{x}$-cross sections were made, (b) X - section at $7.200 \mathrm{~nm}$ of Figure $7 \mathrm{a}$. The blue arrow shows the height of the small red circle of the upper boundaries of the examined platelets' aggregation, (c) X - section at $4.500 \mathrm{~nm}$ of Figure $7 \mathrm{a}$. The red arrow is indicative of the height of the small red circle of the lower boundaries of this platelets' cluster.

of the same platelets aggregation are yellow and this finding shows that they are more electrically positive compared with the nearby areas free of platelets. It can be deduced that the platelets are electrical negative shown by the blue color of their lower boundaries of their central aggregation in the examined EFM image, whereas their upper boundaries are $120 \mathrm{~nm}$ higher compared with the lowest ones. This increase in height is rather too small to be indicative of platelet and may be due to the PRP proteins aggregations such as fibrinogen. It is known that under physiological conditions the overall charge of fibrinogen molecule is negative. However, its $\alpha \mathrm{C}$ domains, which act as a pioneer in the surface binding process, are positively charged (Tebbe et al 2007).

The observed electric current can be explained by two contributing effects: a) as the platelets are not fully dehydrated, there is a fine layer of some water molecules around the platelets which can allow $\mathrm{OH}^{-}$and $\mathrm{H}^{+}$ions to move in the applied electric field, b) polarization effects of water molecules are present.

Figures 8a and b illustrate the AFM topography image of PRP consisting mainly of platelets on biased a-C:H thin film after one hour of incubation and the corresponding EFM image (MAG*COS signal). It can be observed that the areas without PRP (denoted by square) are more electrical positive compared with the areas with platelets as shown with black circles.

The EFM results involving phase and $M A G * C O S$ signal of bare biased and floating a-C:H thin films are shown in Figures 9 and 10. In regards of Biased a-C:H films (a)
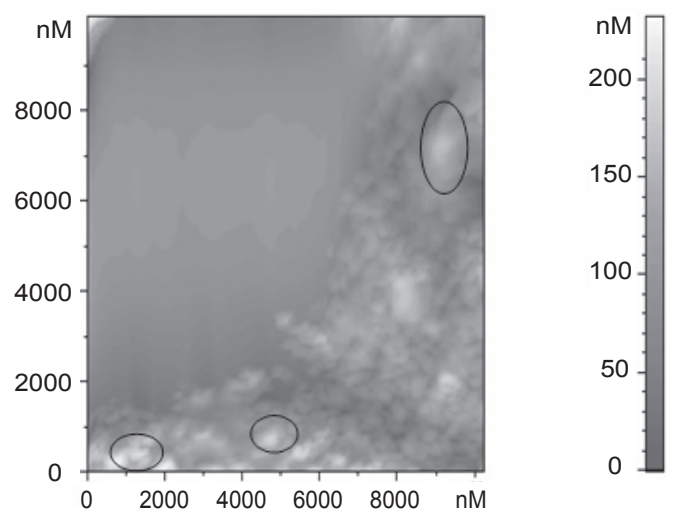

(b)

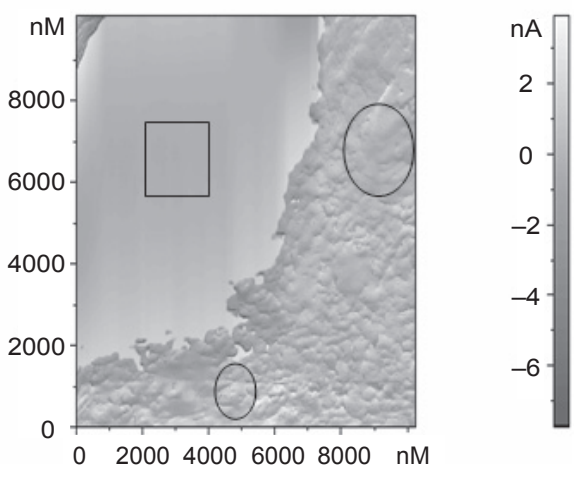

Figure 8 (a) Atomic force microscope topography image of platelets on a-C:H (Biased) after I hour incubation time (scan size $10 \mu \mathrm{m} \times 10 \mu \mathrm{m}$ ). Black circles are indicative of platelets. (b) Electric force microscope (MAG*COS) image of platelets on a-C:H (Floating) after I hour of incubation. The square shows the areas without platelets whereas the black circles indicate platelets. 
(Figures 9a, 9b), the values of $\mathrm{MAG}^{*} \mathrm{COS}$ and phase are equal and higher than $0 \mathrm{nA}$ and indicate that these films are positively charged. As a result, they attract via electrostatic forces the electrically negative platelets increasing their thrombogenicity.

The floating a-C:H thin films exhibit a uniform spatial charge distribution with high flactuations of $\mathrm{MAG} * \mathrm{COS}$ and phase (Figures 10a, 10b). By measuring the average value of $\mathrm{MAG}^{*} \mathrm{COS}(\mathrm{Rm})$ in three different areas A, B, C of Floating a-C:H films, illustrated in Figures $10 \mathrm{a}$, it can be estimated that $\mathrm{Rm}$ values are (-) $0.004 \mathrm{nA},(+) 0.013 \mathrm{nA},(+) 0.002 \mathrm{nA}$, respectively. Thus, the floating a-C:H thin films are mainly electrical positive except for a few areas that are negatively charged. In comparison with the biased a-C:H films, they are less electrical positive than the latter ones explaining partially their decreased thrombogenicity. These floating films compared to the biased ones are more suitable for potential biosensing applications, as they attract more the less negative platelets prone to aggregate. By tailoring the deposition parameters, the control of surface electrical charge of

(a)
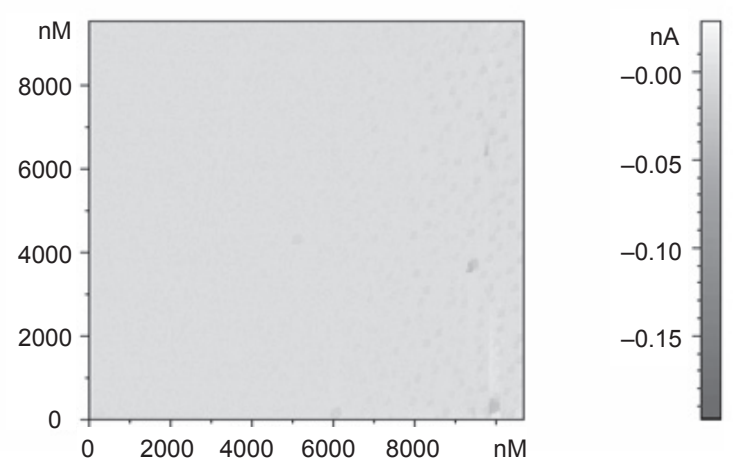

(b)
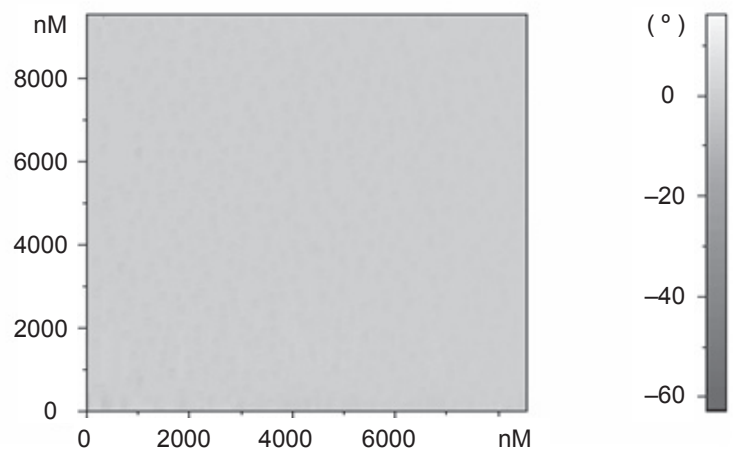

Figure 9 Electric force microscope images of a-C:H (Biased) as deposited (scan size $10 \mu \mathrm{m} \times 10 \mu \mathrm{m})(\mathbf{a}) \mathrm{MAG} * \mathrm{COS}(\mathbf{b})$ Phase. nanomaterials, may be used for the fabrication of biosensors for the detection of platelets with a tendency to form thrombus and cause acute coronary syndromes.

\section{Conclusions}

From the present study, it is verified that the surface morphology and the spatial electrical field distribution influence the thrombogenicity of a-C:H thin films developed by magnetron sputtering. It was found that platelets are electrically negatively charged and variations of the deposition parameters can lead to differently surface charged nanomaterials for potential usage as coatings for cardiovascular applications and as nanosensors for platelets prone to form clot.

The carbon films grown without bias voltage with $20 \% \mathrm{H}_{2}$ in plasma, exhibit less thrombogenicity compared to biased ones. This fact was apparent both qualitatively (from AFM images) and quantitatively (from variations in parameters indicative of surface roughness). A new methodology for the AFM study of platelets response to biomaterials was used and a potential model for platelets aggregation was suggested.

(a)

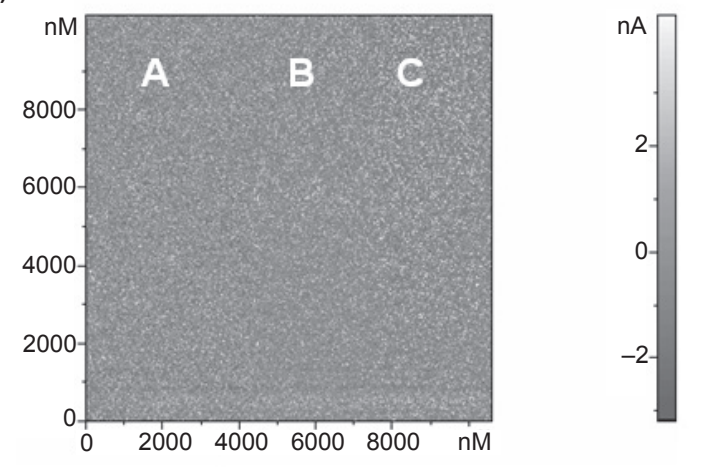

(b)

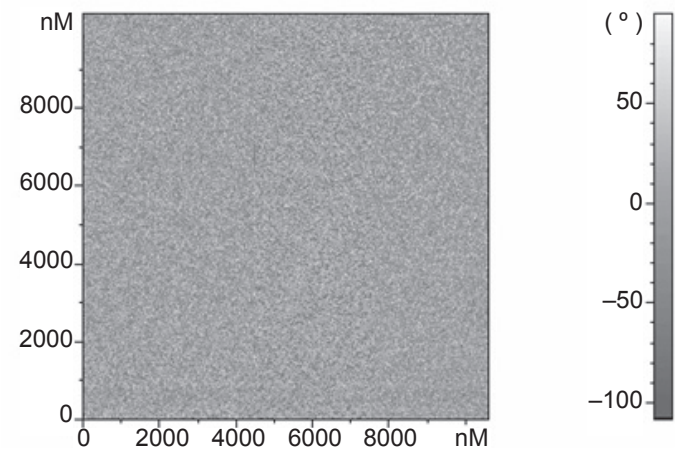

Figure 10 Electric force microscope images of a-C:H (Floating) as deposited (scan size $10 \mu \mathrm{m} \times 10 \mu \mathrm{m})(\mathbf{a})$ MAG*COS (b) Phase. 
The EFM measurements of electrostatic forces distribution during platelets adhesion on biomaterials give information about the contribution of surface positive charges of artificial materials to the attraction of platelets. Thus, the electrical negatively charged biomaterials' surfaces present lower thrombogenicity. Additionally, the more negative thin films, such as floating a-C:H ones compared with the examined biased ones can be potential used for sensing the less negative charged platelets that are prone to form aggregations. As hemocompatibility is determined by a plethora of parameters, besides electrical and topographical properties, the detailed evaluation of other surface properties is needed to make inroads into the vexing problem of biomaterials' failure for biosensing and medical applications.

\section{Disclosure}

The authors report no conflicts of interest in this work.

\section{References}

Bosmann HB. 1972. Platelet adhesiveness and aggregation: II. Surface sialic acid, glycoprotein:N-acetylneuraminic acid transferase, and neuraminidase of human blood platelets. Biochim Biophys Acta, 279:456-74.

Charitidis C, Logothetidis S, Gioti M, et al. 2000. A comparative study of the nanoscratching behavior of amorphous carbon films grown under various deposition conditions. Surf Coat Technol, 125:201-6.

Cui F, Li D. 2000. A review of investigations on biocompatibility of diamond-like carbon and carbon nitride films. Surf Coat Technol, 131:481-7.

Abramson HA. 1928. The electrophoresis of the blood platelets of the horse with reference to their origin and to thrombus formation. $J \operatorname{Exp}$ Med, 47:677-83.

Hai-Tong S, Zheng-Hao L, Zhou J, et al. 2007. An electrostatic force microscope study of Si nanostructures on $\mathrm{Si}\left(\begin{array}{lll}1 & 0 & 0\end{array}\right)$ as a function of post-annealing temperature and time. Appl Surf Sci, 253:6109-12.

Hartwig JH. 2007. The platelet cytoskeleton. In: Michelson AD (ed). Platelets 2nd ed. Amsterdam: Academic Press, pp. 75-97.

Hauert R, Müller U. 2003. An overview on tailored tribological and biological behavior of diamond-like carbon. Diam Relat Materials, 12:171-7.

Hussain M, Siedlecki C. 2004. The platelet integrin alpha(IIb) beta(3) imaged by atomic force microscopy on model surfaces. Micron, 35:565-73.

Kotze HF, van Wyk V, Badenhorst P, et al. 1993. Influence of platelet membrane sialic acid and platelet-associated IgG on aging and sequestration of blood platelets in baboons. Thromb Haemost, 70:676-80.

Kubová O, Švorčík V, Heitz J, et al. 2007. Characterization and cytocompatibility of carbon layers prepared by photo-induced chemical vapor deposition. Thin Solid Films, 515:6765-72.

Laskarakis A, Gravalidis C, Logothetidis S. 2004. FTIR and Vis-FUV real time spectroscopic ellipsometry studies of polymer surface modifications during ion beam bombardment. Nuclear Instrument and Methods in Physics Research Section B: Beam Interaction with Materials and Atoms, 216:131-6.

Liu D, Zhang S, Ong S, et al. 2006. Surface and electron emission properties of hydrogen-free diamond-like carbon films investigated by atomic force microscopy. Mater Sci Eng A, 426:114-20.

Logothetidis S, Gioti M, Lousinian S, et al. 2005. Haemocompatibility studies on carbon-based thin films by ellipsometry. Thin Solid Films, 482:126-32.
Logothetidis S, Gioti M, Patsalas P, et al. 1999. Insights on the deposition mechanism of sputtered amorphous carbon films. Carbon, 37:765-9.

Logothetidis S. 2002. Characterization and spectroscopy of thin films. In: Nalwa HS (ed). Handbook of thin films materials. New York, NY: Academic Press, pp. 277-30.

Logothetidis S. 2007. Haemocompatibility of carbon based thin films. Diam Relat Materials, 16:1847-57.

Lousinian S, Logothetidis S, Laskarakis A, et al. 2007. Haemocompatibility of amorphous hydrogenated carbon thin films, optical properties and adsorption mechanisms of blood plasma proteins. Biomol Eng, 24:107-12.

Lupu C, Calb M. 1988. Changes in the platelet surface charge in rabbits with experimental hypercholesterolemia. Atherosclerosis, 72:77-82.

Maguire P, McLaughlin J, Okpalugo T, et al. 2005. Mechanical stability, corrosion performance and bioresponse of amorphous diamond-like carbon for medical stents and guidewires. Diam Relat Materials, $14: 1277-88$.

Mandic R, Opper C, Krappe J, et al. 2002. Platelet sialic acid as a potential pathogenic factor in coronary heart disease. Thromb Res, 106:137-41.

Massa T, Yang M, Ho J, et al. 2005. Fibrinogen surface distribution correlates to platelet adhesion pattern on fluorinated surface-modified polyetherurethane. Biomaterials, 26:7367-76.

Mitsakakis K, Lousinian S, Logothetidis S, et al. 2007. Early stages of human plasma proteins adsorption probed by atomic force microscope. Biomol Eng, 24:119-24.

[ND-MDT] NT-MDT: Integrated solutions for NanoTechnology. 2008. SPM Principles [online]. Accessed on May 6, 2008. URL: http://www. ntmdt.com/SPM-Techniques/Principles.

Oberprieler N, Roberts W, Graham A, et al. 2007. Inhibition of ADP-induced platelet adhesion to immobilised fibrinogen by nitric oxide: Evidence for c GMP-independent mechanisms. Biochem Pharmacol, 73:1593-1.

Opper C, Clement C, Schwarz H, et al. 1995. Increased number of high sensitive platelets in hypercholesterolemia, cardiovascular diseases, and after incubation with cholesterol. Atherosclerosis, 113:211-7.

Plant S, Grant D, Leach L, et al. 2007. Surface modification of NiTi alloy and human platelet activation under static and flow conditions. Mater Lett, 61:2864-7.

Robertson J. 2002. Diamond-like amorphous carbon. Mater Sci Eng, $37: 129-281$.

Shih CC, Shih CM, Su YY, et al. 2003. Quantitative evaluation of thrombosis by electrochemical methodology. Thromb Research, 11:103-9.

Tebbe D, Thull R, Gbureck U. 2007. Influence of spacer length on heparin coupling efficiency and fibrinogen adsorption of modified titanium surfaces. Biomed Eng, 6:31.

Trip MD, Cats VM, van Capelle FJ, et al. 1990. Platelet hyperreactivity and prognosis in survivors of myocardial infarction. $N$ Engl J Med, 322:1549-54.

Uchihashi T, Ishida T, Komiyama M, et al. 2000. High-resolution imaging of organic monolayers using noncontact AFM. Appl Surf Sci, 157: 244-50.

Vinnichenko M, Gago R, Huang N, et al. 2004. Spectroscopic ellipsometry investigation of amorphous carbon films with different $\mathrm{sp}^{3}$ content: relation with protein adsorption. Thin Solid Films, 455-456:530-4.

Weng KW, Chang CL, Wang DY. 2002. Effect of ion energy on degradation of diamond-like carbon films exposed to high-energy bombardment from an ion implanter. Diam Relat Mater, 11:1447-53.

Yang P, Huang N, Lenge Y, et al. 2003. Activation of platelets adhered on amorphous hydrogenated carbon films synthesized by plasma immersion ion implantation-deposition (PIII-D). Biomaterials, 24:2821-9.

Zhu XD, Ding FH, Naramoto H, et al. 2006. AFM investigation on surface evolution of amorphous carbon during ion-beam-assisted deposition. Appl Surf Sci, 253:1480-3. 
